\title{
Idiopathic Hypersomnia
}

National Cancer Institute

\section{Source}

National Cancer Institute. Idiopathic Hypersomnia. NCI Thesaurus. Code C116343.

A disorder of central nervous system etiology characterized by excessive sleepiness

during the daytime. 